\title{
Agôn
}

Revue des arts de la scène

$4 \mid 2011$

L'objet

\section{Une grammaire de la relation corps-objet}

Entretien réalisé par Émilie Charlet et Aurélie Coulon, le 22 octobre 2011 à Paris

\section{Claire Heggen}

\section{OpenEdition}

Journals

Édition électronique

URL : http://journals.openedition.org/agon/1952

DOI : 10.4000/agon.1952

ISSN : 1961-8581

Éditeur

Association Agôn

Référence électronique

Claire Heggen, «Une grammaire de la relation corps-objet », Agôn [En ligne], 4 | 2011, mis en ligne le 19 décembre 2011, consulté le 15 septembre 2020. URL : http://journals.openedition.org/agon/1952

Ce document a été généré automatiquement le 15 septembre 2020.

Association Agôn et les auteurs des articles 


\section{Une grammaire de la relation corps- objet}

Entretien réalisé par Émilie Charlet et Aurélie Coulon, le 22 octobre 2011 à Paris

\section{Claire Heggen}

1 Claire Heggen est interprète et metteur en scène. Sa pratique est hybride, à la croisée des techniques du mime, de la danse et du théâtre de marionnettes. Elle dirige avec Yves Marc le Théâtre du Mouvement ${ }^{1}$, structure qui a la particularité d'être vouée à la fois à la création et à la transmission. Elle enseigne également à l'École Supérieure Nationale des Arts de la Marionnette.

De cette articulation entre création et pédagogie, est née une grammaire de la relation corps-objet dont elle nous a exposé les fondamentaux ${ }^{2}$.

\section{L'objet à la croisée des pratiques}

ÉMILIE CHARLET. Votre pratique est hybride : ce n'est pas tout à fait de la danse, pas tout à fait du théâtre, pas vraiment du théâtre de marionnettes. La question de l'objet nous semble se situer au carrefour de différentes pratiques. Comment avez-vous envisagé l'objet, au cours de votre formation, de votre histoire et de votre pratique, par rapport à cette catégorisation des arts?

c. H. Pour moi, le rapport à l'objet remonte à très loin, mais il n'est pas passé directement par l'objet marionnettique. J'ai eu une formation de danseuse classique, mais à l'époque il n'y avait que l'Opéra et je ne faisais pas partie des sylphides recommandables. Je me suis ensuite orientée vers le professorat d'éducation physique, et j'ai été reçue au concours d'entrée à l'ENSEP, l'École Normale Supérieure d'Éducation Physique, qui n'existe plus aujourd'hui. Dans les pratiques sportives, le rapport à l'objet est très divers : on va le pousser, le tirer, sauter au-dessus, se plier autour. Quasiment toutes les pratiques sportives sont en relation avec un objet: on est sur une poutre, on tourne autour d'une barre asymétrique, on rebondit sur un tremplin pour passer un mouton, on saute des haies, on lance un javelot, ou bien on 
glisse sur la neige avec des skis ou on borde une voile. À cette époque, c'était le tout début de la gymnastique artistique avec objets: on commençait à utiliser des massues, des rubans, des cordes, des cerceaux ou des bâtons. La discipline sportive olympique n'était pas encore élaborée, loin de là. Et puis, toujours dans le cadre de cette formation, j'ai également pratiqué les techniques somatiques, c'est-à-dire toutes les techniques de prise de conscience du corps dans le mouvement, entre autres d'après le travail de Gerda Alexander. On travaillait beaucoup le toucher et le contact avec des petites balles, des bambous de tailles différentes, des marrons. C'était intéressant pour moi de reprendre ensuite ces techniques dans le cadre de la formation des marionnettistes.

C'est un premier moment de ma formation, mais ma passion restait la danse : je suis allée me former aux Etats-Unis, et en France j'ai expérimenté tout ce que je pouvais, Baratha Natyam, Nô, kabuki, claquettes, jazz, mais aussi le théâtre d'actions physiques de Grotowski. J'ai travaillé avec le professeur de danse de l'ENSEP, Monique Bertrand, alias Pinok, qui constituait un duo avec Matho, une autre femme. Elles développaient tout un mouvement d'expression corporelle à base de mime corporel. Elles avaient travaillé avec Étienne Decroux ${ }^{3}$, le maître du mime contemporain. Tout naturellement, une fois enseignante, je suis allée travailler tous les jours chez lui pendant quatre ans et demi. Très rapidement, j'ai été appelée à enseigner la danse dans l'école dans laquelle j'avais été formée: je n'ai pas été longtemps professeur d'éducation physique en collège. Très vite, j'ai commencé à créer. Puis j'ai été détachée à la Fédération Française de Danse pendant un an, ensuite j'ai démissionné. J'avais rencontré un partenaire, Yves Marc, qui est devenu mon mari, et nous avons développé ensemble la compagnie.

Étienne Decroux se revendiquait très fortement de Craig, il y a d'ailleurs une correspondance entre eux dont j'ai pu prendre connaissance. J'ai compris a posteriori sa perspective. Dans le travail de Decroux, l'acteur est sujet-objet d'art, c'est-à-dire qu'il doit montrer son art sans montrer sa personne, ce qui pourrait déjà être une définition du marionnettiste et de la marionnette. Nous avons navigué sur cette hypothèse-là, sur cette utopie du mime corporel et dramatique, non pas dans son esthétique mais dans ses fondamentaux. Ceux-ci fonctionnent pour toutes les pratiques: pour les acteurs, pour les danseurs, pour les marionnettistes, et pour la marionnette elle-même. C'est un travail du regard, de la posture, de la respiration, de la segmentation, de l'organisation dans l'espace, de l'orientation. L'énigme de la surmarionnette de Craig a alimenté l'utopie du mime corporel et dramatique de Decroux, et nous avons été porteurs de cette utopie à travers nos différentes créations.

Au début, juste après notre formation chez Decroux, nos corps étaient objets, sujetsobjets d'art, ils étaient objectivés par le collant qui nous recouvrait: c'était des figures. Nos personnes n'étaient pas en jeu. À l'époque à Paris il y avait Lecoq, Decroux et Marceau. Sortant de chez Decroux, nous n'avions pas pour autant l'intention de reproduire son style. Pour nos créations, nous avons toujours travaillé avec des objets. Ces objets n'étaient pas des accessoires, mais des déclencheurs et des attracteurs dramaturgiques. C'était la circulation de l'objet qui faisait l'histoire. On ne cherchait pas à raconter une histoire, il n'y avait pas de scénario a priori: on travaillait sur des formes, des dynamiques, des thématiques et à partir de là un matériau, une esthétique, un vocabulaire, une syntaxe se dégageaient. On développait 
une esthétique différente à chaque création. À partir de 1975, on a travaillé sur des formes animées de l'intérieur, sur l'objet, sur le masque, le déplacement de masques sur le corps, la musicalité, l'animalité, mais aussi la marche humaine, le portrait corporel, la pensée de l'acteur, les états émotionnels de l'acteur... nous étions des transversaux avant la lettre, des précurseurs de ce qui se passe maintenant, et je le dis sans prétention : nos spectacles en témoignent, c'est concret, ça existe.

\section{La construction d'une grammaire}

C. H. Puis j'ai été invitée il y a vingt-trois ans par Margareta Niculescu à l'ESNAM, à Charleville-Mézières, pour faire un training pour l'acteur marionnettiste. Tout de suite, je me suis dit qu'il fallait travailler d'emblée avec des objets et imaginer le training du marionnettiste à partir de l'objet. J'ai apporté des petits bambous, des draps, du papier de soie, de petits cadres, de grands bâtons, des choses assez simples, mais qui m'ont permis de dégager toute une grammaire de la relation corps-objet pour les étudiants marionnettistes. A l'époque, la manipulation à vue s'imposait, or, si on manipule à vue, le corps ne peut pas être investi de la même façon suivant le mode de manipulation, d'autant plus que chaque marionnette demande une organisation différente des corps selon qu'on la manipule seul, à deux, à trois, etc. Cela faisait apparaître des modes d'énonciation de l'acteur très différents : si l'acteur est en scène en même temps que sa marionnette, qui est-il ? Est-il associé à sa marionnette, présent-absent, pour manipuler dans un espace visuel contraint à l'amplitude de la main, ou est-il dissocié dans l'espace? Le choix de la dissociation implique des déplacements, la prise en compte de la hauteur, de la profondeur et l'occupation d'un espace beaucoup plus large. J'ai aussi enseigné à toutes les promotions ce travail du déplacement des masques sur le corps. Le masque ${ }^{4}$ a un certain fonctionnement, une facialité, un regard, une pensée et le corps s'organise en fonction du masque. Mais si on déplace le masque pour le mettre à l'épaule, par exemple, quels sont les petits corps qui apparaissent? Un corps nouveau, plus petit, avec d'autres propriétés articulatoires naît des mouvements du bras : cela peut être un personnage aquatique ou aérien si je fais onduler mon bras, ou un personnage très terrestre si je l'ancre sur une surface. Le terme de " corps-castelet » a fait florès avec Alain Recoing, puis avec Nicolas Gousseff. Decroux appelait ça le «corps géographique ", nous parlons de «scénographie mouvante». À partir de ces différentes données, j'ai développé un training et une grammaire de la relation. Margareta Niculescu voulait aussi que je suive les projets des étudiants, j'ai donc travaillé avec eux sur la manipulation des marionnettes et sur les dramaturgies qui se mettaient en route.

É. c. L'une des questions qui nous occupe est justement l'écriture de l'objet. Comment est-ce que l'objet permet d'écrire un spectacle sans texte, sans vouloir a priori raconter une histoire?

C. H. Je peux parler à ce sujet d'une pièce que j'ai mise en scène à partir des partitions de Georges Aperghis. Nous avons travaillé avec lui lors d'un stage de théâtre musical à Aix-en-Provence. Il y avait une majorité de musiciens, et nous, nous proposions des interprétations des textes avec du mouvement. Nous avons eu envie de prolonger ce travail, et il nous a laissé les partitions avec lesquelles on avait travaillé, c'est-à-dire par exemple Enumération, Conversation, Récitations. Nous avons essayé de mettre en relation la musicalité du mouvement et la musicalité des partitions, mais au début ce 
n'était pas forcément utilisable pour créer un spectacle. Et un jour, en arrivant dans le gymnase où on répétait, on a trouvé des caisses de tailles différentes qui avaient servi à former un podium lors d'une rencontre de judo. Nous avons travaillé une journée entière avec ces caisses, en tournant autour, en entrant à l'intérieur, en les déplaçant, et nous avons décidé de les garder : c'est finalement la relation avec les caisses qui a fait sens. Le corps est formalisé par les caisses, quand il se plie pour entrer à l'intérieur, ou quand il s'organise pour les porter, car elles étaient très lourdes. Nous étions obligés de tourner autour de ces cubes, et nous cherchions comment passer d'une caisse à l'autre, comment passer dessus : des questions très simples se posaient. Finalement, j'ai demandé aux acteurs une gestuelle cubiste, à la Decroux : comme on travaillait sur des caisses carrées, il a fallu développer une gestuelle non réaliste, et tout le jeu a été de passer d'un corps à effet de réel à un corps formalisé, avec des retours réguliers à l'effet de réel. Il y a eu six ou sept scénarios différents, avant qu'on arrive au scénario définitif, Encore une heure si courte, qui laissait tout de même la porte ouverte à des trouvailles. On l'a créé en 1989 et je l'ai remonté cette année.

C'est à ce moment-là que j'ai été invitée à enseigner à Charleville : je mettais en place une grammaire pour mes acteurs, d'une manière vraiment spécifique car avant nous avons toujours utilisé des objets, mais pas de cette manière-là. Il y a ce qui est de l'ordre de la manipulation, et ce qui est de l'ordre du symbolique. À partir de Decroux, nous avons fait tout un travail de corps métaphorique, de corps extraquotidien. Les objets devenaient eux aussi métaphoriques. Les caisses peuvent devenir un bateau, le ventre d'une prison, une décharge, un bureau, une tempête. L'écriture se fait à partir de cette conjonction entre la manipulation et le jeu métaphorique. C'est l'articulation entre ces différentes métaphores, à l'aide du travail technique de manipulation, qui fait le sens. On associe des éléments, on bricole, on essaye différents scénarios. Certaines choses se fixent, mais nous sommes toujours en train de remettre sur le métier notre ouvrage, et quand on risque de s'enfermer trop dans un sens, on introduit des variations qui peuvent être rythmiques, spatiales, corporelles ou symboliques. C'est du théâtre qui s'écrit en se faisant, et le scénario arrive a posteriori. Le chemin pour y arriver est très différent de celui que prennent ceux qui ont un scénario de départ, et cherchent ensuite à y adapter des formes. Il faut qu'il y ait différentes lectures possibles pour l'acteur, pour le metteur en scène et pour le spectateur.

A.c. Pour Encore une heure si courte, vous avez donc inventé une gestuelle spécifique, adaptée à ce travail avec les boîtes. Est-ce que vous diriez que chaque nouveau matériau, chaque type d'objet et chaque scénographie induit une technique corporelle différente?

C. H. Oui, c'est ça. Je prends un autre exemple : j'ai fait tout un travail de recherche sur la marche humaine $e^{5}$, et il a fallu quatre ans de recherches avant de présenter le premier spectacle. Pendant deux ans, j'ai travaillé pour moi, j'ai observé, je me suis servie des cours d'anatomie et de physiologie que j'avais eus lors de ma formation en éducation physique. Je me suis aussi appuyée sur le travail de Decroux. Nous avons animé des stages conjoints sur la marche avec Blandine Calais, qui était kinésithérapeute ${ }^{6}$. Elle se concentrait sur les questions d'anatomie et de physiologie, et je cherchais à créer un vocabulaire dramatique à partir de la marche. La moindre petite transformation à un niveau articulaire retentit sur l'ensemble du corps et induit des états de corps, des états de tension, des états de relâchement qui font que 
des caractères apparaissent. Il s'agissait de décrypter les démarches individuelles et leurs tendances, puis d'abstraire ces tendances pour y inscrire d'autres caractères. Petit à petit, j'ai développé cette recherche avec la compagnie. On faisait émerger une grammaire, et je formais aussi les acteurs de la compagnie pour qu'ils puissent la transmettre. C'est une dimension fondamentale pour la compagnie: la création, donne lieu à de la diffusion, de l'enseignement, de l'action culturelle. Notre passé de pédagogues n'était pas séparé de notre travail de création. Aujourd'hui, la transmission est devenue une des spécificités de la compagnie.

\section{Décomposition, recomposition, métaphore}

A. c. Chez Decroux, lorsqu'un objet virtuel intervient dans un enchaînement, ce n'est jamais sur le mode figuratif. Si on prend l'exemple de l'enchaînement du «verre d'eau », il est impossible de boire en faisant le geste inscrit dans cette variation. Comment en êtes-vous venue à réutiliser des objets concrets en gardant cette valeur métaphorique de l'objet, déjà présente chez Decroux?

C. H. Picasso est venu voir le travail de Decroux et lui a dit : " On fait le même travail ». Decroux ne s'intéressait pas à l'anecdote, contrairement à d'autres mimes. Il disait qu'il ne voulait pas épater la concierge. Il cherchait à élaborer un art d'acteur en travaillant sur la décomposition-recomposition des actions, du corps, de l'espace, de la dynamique du temps. Il ne faut pas oublier qu'il a été apprenti boucher dans sa jeunesse, puis brancardier dans les tranchées pendant la première guerre mondiale, à l'âge de dix-sept ans. L'anatomie, il savait ce que c'était. Dans Paroles sur le mime il écrit : «j'incise des frontières ». Ce n'est pas anodin. Le corps est segmenté, les yeux, la tête, le cou, la poitrine... correspondent à différents segments. Les actions sont elles aussi décomposées en fonction d'un mode articulaire. Decroux se disait ossiste, du mot « os ». L'espace est décomposable en trois dimensions, le temps est segmenté. Il était à la fois cubiste et impressionniste, et on retrouve dans son travail des traits du théâtre japonais. Par exemple, pour regarder vers le haut à gauche, on décompose le mouvement en trois temps, rotation puis inclinaison latérale enfin inclinaison vers l'arrière pour terminer par une pause, on donne ainsi plus d'importance au point final du regard. Dans le théâtre japonais aussi, il est préconisé de faire passer le regard par trois points. A partir d'un trajet indirect de la tête de l'acteur, l'œil du spectateur reconstitue le trajet direct du regard, comme lorsqu'on recrée du vert en voyant une plaque de bleu et une plaque de jaune côte à côte sur un tableau impressionniste. C'est de l'anthropologie théâtrale, et d'ailleurs Eugenio Barba s'en est beaucoup inspiré.

Ce sont des fondamentaux, donc forcément on travaille avec. J'ai tout de suite abordé ça avec les étudiants de Charleville, en leur donnant le vocabulaire commun nécessaire. D'une promotion à l'autre, ils savent ce qu'est un mouvement progressif, et comment concentrer l'attention du spectateur sur l'objet ou sur l'acteur. Comment amener le regard du spectateur vers ce qu'on veut qu'il regarde ? Cela vient aussi du théâtre japonais : l'acteur est le maître du regard. 
É.c. On a l'impression en vous écoutant que l'objet, dans ce rapport de décompositionrecomposition du mouvement, est à la fois une contrainte et un point d'appui pour déconstruire et reconstruire une progression.

C.H. Oui, c'est tout à fait ça. La géométrie en elle-même est déjà expressive, la chronologie aussi. Cela me permet de constituer une partition gestuelle, corporelle. Ce que voit le spectateur est plus large que le fait de coller à un sens. Ce n'est pas de la psychologie. Le fonctionnement de la marionnette est du même ordre.

É.c. Est-ce que chaque type d'objet induit une forme d'improvisation spécifique? Entre la boîte et le masque, il y a un monde.

C. H. Oui, il y a un monde, ce n'est pas le même objet mais les règles de fonctionnement sont les mêmes. Il y a une base commune. Comme le masque, ma boîte peut penser, elle peut regarder si je la manipule d'une certaine manière.

É. c. Ça a un lien, me semble-t-il avec la notion de prothèse, qu'on rencontre souvent quand on travaille sur l'objet, par exemple chez Kantor. II est difficile d'aller au-delà de ce constat, et de mieux cerner cette notion dans le champ des arts scéniques. En vous écoutant, on perçoit mieux ce lien entre l'animé et l'inanimé, qui est diffıcile à saisir.

C. H. Pour moi, l'objet, ce n'est ni l'objet ni le sujet, mais la relation des deux. J'emploie le mot de relation aussi au sens de « relater une histoire ». L'objet en tant que tel va me donner des informations sur l'état dans lequel il est. S'il est lourd, il va avoir un effet sur mon centre de gravité. Si je l'écarte un peu de mon corps, il va m'entraîner. Le sujet et l'objet ne sont donc pas séparés : comme dirait Françoise Dolto, c'est du coêtre, de la coexistence. L'objet me donne des informations, par la vue mais aussi par le toucher. Est-ce qu'il est lourd ? Est-ce qu'il bouge ? En m'informant, il me transforme, et cela devient performance. J'ai trouvé une série de termes en anglais qui exprime bien cela : informance, transformance, performance. On est à la fois dans l'attention, dans l'intention et dans la tension. C'est ce jeu permanent entre l'objet et le manipulateur qui fait la présence et le sens. Mon objet peut m'indiquer une direction alors que j'en aurais proposé une autre. C'est la matérialité de l'objet qui réajuste et relance en permanence le sens qui est en train de se produire.

É. c. Est-ce qu'il y a des objets plus dociles que d'autres, plus faciles à appréhender?

C. H. C'est très personnel. Quand on commence à travailler avec des bambous, et qu'on les reprend le lendemain, les étudiants ou les stagiaires reconnaissant «leur» bambou. Certains vont adorer le papier de soie. Il y a des gens qui détestent le drap, d'autres qui se vautrent dedans pendant des heures. Une marionnette aussi, qu'elle soit réaliste ou que ce soit un bout de mousse, c'est la poupée qu'on traîne. À un moment donné, la notion d'attachement entre en jeu. À partir du moment où on commence à travailler avec un objet, un passé commun se crée.

C'est très important pour moi de faire la différence entre accessoire et objet. L'objet est ce qui est " posé devant », le sujet est " assujetti à ». On est assujetti à l'objet qui est posé devant. Quel est le trajet intérieur de l'objet? Chacun informe l'autre, on cherche comment travailler avec la gravité, avec l'inertie de l'objet. Le toucher n'est pas le contact: quand je touche le bout de mon crayon, j'entre en contact avec la table. Quand je manipule la marionnette, je touche un endroit mais projette dans le regard ou dans le nez de la marionnette pour aller toucher le spectateur à l'autre bout de la salle. Je ne manipule pas là où je touche. C'est à cela qu'on reconnaît les bons et les mauvais marionnettistes. 


\section{Inventaire : objets, marionnettes, matériaux}

A. c. Vous nous avez raconté que vous avez travaillé avec des caisses trouvées dans une salle de sport. Est-ce qu'il vous arrive de fabriquer ou de faire fabriquer des objets pour vos spectacles?

C. H. Dans mon dernier solo, Les choses étant ce qu'elles sont, tout va aussi bien que possible, qui est beaucoup plus marionnettique que le précédent, j'utilise des galets, un grand manteau d'homme et un deuxième manteau, qui est le double du premier mais qui est en feutre, dont va sortir un personnage-oiseau. Il y a de grands voiles qui se transforment et des marionnettes non réalistes : une marionnette plate faite avec du fil d'aluminium recuit et recouverte de non tissé blanc qui a une forme humaine proche de ma taille, que je manipule avec tout mon corps; une marionnette en forme de boule que je fais rouler sur mon corps et que je déplie ensuite, avec laquelle j'instaure un jeu de relation. Celle-ci est plus proche de la marionnette que la précédente. Il y a aussi un fil de près de cinq mètres que je construis sur mon corps et que je manipule dans l'espace. C'est la pièce la plus marionnettique. Mais par exemple pour "Tezirzek", la pièce qui a été filmée pour la télévision, on a fait construire de grandes statues habitées de l'intérieur qui se déplacent dans le désert. Quand on a eu l'idée de se mettre des masques sur le corps, on nous a dit qu'on faisait de la marionnette alors que pour nous, c'était du mime corporel. Pour nous, ça découlait complètement du système decrousien. Dans notre tout premier spectacle, avant même d'aller chez Decroux, on jouait deux marionnettes. On faisait une hydre à dix têtes avec des masques. Il y avait une femme-boule et un homme-cube qui se rencontraient et qui copulaient. La boule accouchait d'un fotus rouge dans lequel on s'enveloppait, qu'on faisait gonfler et qui explosait ensuite. Dans notre premier spectacle il y avait presque toutes les thématiques qu'on a abordées ensuite: l'animalité, les formes animées, le déplacement des masques sur le corps. Dans le prochain spectacle, il y aura des marionnettes plus marionnettiques encore : je vais reprendre la marionnette plate et la marionnette-fil et je vais les développer davantage.

Éc. En voyant cette typologie d'objets qui fait votre parcours, on peut se demander jusqu'où l'objet peut être informe, notamment quand vous parlez de ce fil que vous vous enroulez autour du corps. Est-ce que l'objet doit nécessairement avoir une forme, ou est-ce qu'on peut en élargir la définition?

C. H. Je me situe plutôt dans cette direction-là à travers la dimension du jeu métaphorique avec les matériaux. J'ai monté en 1999 une pièce qui s'appelle Cities, inspirée des Villes invisibles d'Italo Calvino. J'ai travaillé dans cinq villes d'Europe différentes, Oslo, Bratislava, Amsterdam, Madrid et Paris. Dans chaque ville, je suis partie quinze jours faire un travail de formation élémentaire auprès de jeunes professionnels et les amener à élaborer en même temps une écriture individuelle sur la mémoire de leur ville, en choisissant avec eux un objet qui pouvait représenter leur ville. Ensuite, j'en ai sélectionné une quinzaine. Ils sont venus dans le Cantal, dans un lieu qu'on programme l'été, et on a de nouveau travaillé quinze jours. Ils ont $\mathrm{pu}$ communiquer directement car ils avaient une grammaire commune, et ils apprenaient les villes des autres. C'était très intéressant, socialement et culturellement: la gestuelle des femmes espagnoles n'a rien à voir avec la gestuelle des nordiques. Ensuite, j'en ai sélectionné sept avec qui j'ai monté le spectacle, qui a tourné dans les cinq villes concernées. À Bratislava, c'était la période de la 
restitution: les maisons avaient été réquisitionnées, et après la chute du mur les biens réquisitionnés ont été restitués à leurs propriétaires. Parallèlement, on reconstruisait le centre-ville où se côtoyaient des maisons en ruines et des bâtiments neufs, très pimpants et colorés. Les éléments qu'ils ont apportés étaient des matériaux à travers lesquels on pouvait voir, mais qu'on ne pouvait pas traverser : l'arrière d'une machine à laver, par exemple. À Oslo ils avaient choisi des vitres, du verre et des miroirs. C'était la glace, ce qui correspond métaphoriquement à leur relation à l'autre. Madrid, c'était une porte avec des petits carreaux, dont un manquait. Or, c'est une ville où il y a plusieurs épaisseurs de souterrains: on retrouvait cette idée du passage, de la traversée. Pour Amsterdam, on a travaillé avec du papier de soie très léger, et pour Paris avec des planches.

J'ai repris une partie de ce travail pour une pièce, Le Petit Cépou, une sorte de petit poucet des banlieues, à partir d'un texte de Pepito Matteo, qui est conteur. J'ai utilisé les matériaux de manière métaphorique : il y a des planches, des vitres, des bâtons, des draps, des ombres et de gros rouleaux pour enrouler des fils électriques que j'ai trouvés au coin de la rue. Ces rouleaux deviennent tour à tour, le labyrinthe dans lequel se perd le petit Cépou, puis la marmite de l'ogre, le ventre de la femme de l'ogre, un refuge, etc.

É. c. Votre travail me semble pouvoir être mis en rapport avec la notion d'absurde, qui entre en jeu par exemple dans Encore une heure si courte. Cela se retrouve aussi dans d'autres spectacles : est-ce que l'objet est lié à la notion d'absurde ? Comment vous emparez-vous de cette notion, qu'on associe spontanément plutôt au théâtre, avec les moyens du mime et de la danse?

C.H. Nous n'avons jamais travaillé directement sur la notion d'absurde. Mais le traitement corporel, par exemple la gestuelle cubiste pour Encore une heure si courte, est absurde. Cela ne provoque pas un franc comique, mais plutôt de l'humour. Les situations arrivent de manière absurde, mais ce n'est pas une chose qu'on cherche particulièrement. J'ai l'impression que l'absurde vient plutôt du détournement d'un objet manufacturé. Or le théâtre d'objets, c'est encore autre chose, cela vient des arts plastiques. Je pense à Roland Schön, par exemple. Les problématiques sont différentes.

Il y a une dimension de l'absurde qui peut venir des associations paradoxales, de la rupture de l'enchaînement causal ou psychologique. Philippe Genty fait ce genre de choses, d'ailleurs il travaille beaucoup sur la psychanalyse. Il y a une logique absurde d'apparitions, de disparitions, d'escamotages.

É. c. Chez Beckett, qui vient immédiatement à l'esprit quand on parle d'absurde au théâtre, il y a l'idée d'épuiser l'objet, de l'user jusqu'à la corde. On peut aussi observer cette tendance dans le mime et dans la danse, mais a priori pas du tout dans le théâtre d'objets.

C. H. Le théâtre d'objets a une autre dramaturgie, qui est plus proche de la narration. Il y a un cousinage évident entre le monde de la marionnette et le monde du mime corporel, d'abord en raison de la référence à Craig. Et puis, à partir du moment où on travaille avec une marionnette anthropomorphe, on travaille sur la segmentation, les articulations. Ce sont des fonctionnements communs: on essaye d'amener le corps humain à des fonctionnements extra-quotidiens, alors qu'on tente d'amener la marionnette vers du quotidien.

Mais quand on travaille sur les transformations de matériaux, c'est encore un autre fonctionnement. Chaque matériau indique de quelle manière on doit le manipuler, et 
crée ainsi des univers différents. Par exemple, quand j'ai travaillé sur Cities, j'avais trouvé un certain type de papier de soie qui nous avait permis d'écrire toute une séquence. Puis, il s'est trouvé que le papier de soie était épuisé. On en a trouvé un autre, beaucoup plus léger. Il a fallu réécrire la séquence, car ça ne marchait plus, les durées n'étaient pas les mêmes, ça ne racontait plus la même chose. L'objet n'a pas d'états d'âme : son vocabulaire, c'est la gravité, le poids.

\section{Corporéité de l'objet, objectivation du corps}

A. c. Vous avez employé le terme de grammaire: est-ce que vous travaillez à partir de la kinesphère ${ }^{7}$ de Laban ? Est-ce que cela fait partie de votre grammaire?

C. H. Oui, bien sûr, c'est fondamental et cela fait aussi partie de manière élémentaire de la formation de base des étudiants marionnettistes. Je travaille régulièrement làdessus: trop souvent, les manipulateurs se placent face public, dans l'axe de la marionnette, et je lutte contre cette tendance.

A. c. En vous écoutant, je me disais qu'on pourrait penser non seulement la position du corps dans l'espace, mais aussi la «posture » et les déplacements de l'objet à partir de la kinesphère.

C. H. Quand je parle du corps, je parle de l'objet. Un trajet d'un point à un autre n'est pas nécessairement rectiligne ou direct: on peut faire des variations de hauteur, d'orientation du corps, de décomposition de l'espace. Plus je me rapproche, plus je diffère le moment d'arrivée, par exemple. J'ai aussi travaillé sur la notion de proxémie. L'espace intracorporel du corps du manipulateur est l'espace intercadre de la marionnette, selon le terme decrousien.

É.c. Est-ce qu'on peut considérer que l'objet crée un dispositif? Est-ce que l'objet est toujours-déjà là sur la scène, est-ce un dispositif à la base du travail, auquel s'ajoute une technique corporelle?

C. H. La technique ne vient pas «en plus ». Les deux coexistent, l'objet et le travail corporel sont en relation, et c'est cette relation qui m'intéresse. Le masque m'intéresse parce qu'il est précisément l'interface entre le marionnettiste et le mime. Si je fais disparaître le masque, et si je joue comme s'il était encore là, avec son empreinte en creux, je suis dans le mime corporel : j'ai incorporé la contradiction à l'intérieur du corps. Si je le délègue au bout d'un segment, un pied par exemple, en le mettant à distance du corps, j'arrive à la marionnette.

É. c. Comment procédez-vous pour garder en mémoire un spectacle au moment de la création? Est-ce que vous prenez des notes?

c. H. Je garde des traces vidéo, mais je m'en méfie beaucoup: il n'y a pas de profondeur, cela modifie la perception des durées. J'utilise des dessins, je prends des notes en utilisant le vocabulaire decrousien. Par exemple : " un progressif de tête en inclinaison latérale en décéléré ", pour moi, c'est clair. C'est toujours à préciser bien sûr, et c'est tout de même la mémoire du corps de l'acteur, la mémoire cellulaire qui prime. Même si on n'a pas joué un spectacle pendant vingt ans, on peut le retrouver.

A. c. Le rythme et la musicalité semblent avoir une place importante dans votre travail. Une caisse peut servir de percussion, par exemple. Est-ce que l'objet se prête particulièrement 
au travail du rythme ? Est-ce que certains objets, comme les caisses, s'y prêtent mieux que d'autres?

C. H. Après la fin de notre formation, nous sommes retournés régulièrement voir Decroux et un jour il nous a demandé : « En tant que mimes professionnels, pourriezvous me dire quelle sorte de mimes vous faites ? À ce moment-là, on travaillait sur un spectacle qui s'appelait Equilibre instable, inspiré d'une exposition de Paul Klee qui nous avait fascinés. Paul Klee était aussi violoniste, c'était un excellent musicien. Nous avons parlé à Decroux de ce spectacle, structuré de rebonds d'une partie du corps sur une autre, qui va elle-même rebondir sur le corps de l'autre : c'était un jeu de causalité d'un corps à l'autre. Et il nous a dit: "Je vois, vous faites du mime musical ». En quatre ans et demi de formation chez Decroux, je ne l'avais jamais entendu parler de mime musical, et pour nous ça a été un déclic. Dès ce moment-là, nous avons toujours été très attentifs à cette dimension de la musicalité. Encore une heure si courte fait partie des pièces explorant cette thématique, et c'est d'ailleurs pour cette raison qu'on était allés travailler avec Georges Aperghis. Ce qui m'intéresse, c'est en fait la relation des musicalités entre elles. La marionnette, le matériau, le corps ont chacun leur propre musicalité.

A. c. Nous avons remarqué au cours de notre enquête que l'objet, pourtant non animé, est souvent propre à créer l'illusion chez le spectateur. Paradoxalement, on a tendance à projeter les caractéristiques du vivant dans un objet inerte.

C.H. La marionnette suscite une fascination pour la petite personne. C'est une des questions qui se sont posées au cours de mon travail, à propos de la manipulation à vue : les spectateurs ont envie de s'illusionner. À partir de là, comment travailler la présence-absence du manipulateur, dans le lieu même de son effacement? Ma réponse a été l'organicité. Si je suis sur le chemin de ma marionnette, je suis obligée de m'organiser pour la laisser passer. C'est le corps qui se plie aux désirs de l'objet. Deux objets d'art sont présents en scène. On donne à voir la relation d'un acteur sujet-objet d'art avec un objet en attente de devenir sujet d'art. A partir du moment où il devient sujet d'art, je cherche à m'objectiver pour me mettre à son service. C'est une grammaire fondamentale, mais cela peut devenir une esthétique. Et cela devient, de toute façon, un entraînement corporel de l'acteur marionnettiste. Cet entraînement est technique, il demande un engagement du corps, et il est aussi sensible car il produit du sens. L'association de mes états de corps et de la signification produite émerge de cette relation entre le corps et l'objet.

\section{NOTES}

1. Le site internet de la compagnie est accessible à l'adresse suivante: http:// www.theatredumouvement.com. À ce sujet, nous vous invitons aussi à lire un entretien dans lequel Claire Heggen évoque la pratique hybride de la compagnie : voir « Théâtre du mouvement : entretien avec Philippe Ivernel ", in Théâtre et danse : un croisement moderne et contemporain, vol. II Paroles de créateurs et regards extérieurs, Études théâtrales n49, Louvain, Centre d'études théâtrales, 2010, p. 113-116. 
2. Concernant la relation corps-objet, voir aussi: Claire Heggen, «Sujet-objet: relations et pourparlers ", in Alternatives théâtrales $n^{\circ} 80$, Objet-danse, $4^{\mathrm{e}}$ trimestre 2003, p. 32-36.

3. À ce sujet, nous vous invitons à consulter l'ouvrage d'Étienne Decroux, Paroles sur le mime, Paris, Librairie théâtrale, 1963. Voir aussi l'ouvrage dirigé par Patrick Pezin : Étienne Decroux, mime corporel, Saint-Jean-de-Védas, L'Entretemps, 2003.

4. À ce sujet, vous pouvez vous référer à l'ouvrage dirigé par Odette Aslan et Denis Bablet : Le Masque, du rite au théâtre, Paris, éditions du CNRS, 2005.

5. Voir l'article intitulé « la marche et l'acteur » qui figure dans l'ouvrage dirigé par Odette Aslan, Le Corps en jeu, Paris, éditions du CNRS, 1993.

6. Blandine Calais est également l'auteur de l'ouvrage Anatomie pour le mouvement, [tome 1: Introduction à l'analyse des techniques corporelles; tome 2: Base d'exercices] Méolans-Revel, éditions Désiris, 1990.

7. Il s'agit de la sphère imaginaire qui entoure le corps et définit l'espace du mouvement tel qu'il peut se déployer autour de l'individu. La kinesphere sert de base au système de notation du mouvement élaboré par Rudolf Laban. «La kinesphère est la sphère autour du corps dont la périphérie peut être atteinte par les membres aisément allongés sans que le corps sur un seul pied ne se déplace du point de support». Rudolf Laban, cité par Simon Hecquet et Sabine Prokhoris in Fabriques de la danse, Paris, PUF, 2007, p. 57.

\section{INDEX}

Mots-clés : Théâtre du Mouvement, ESNAM, ENSEP, Decroux (Étienne) 